\title{
Análise do Perfil Alimentar e do índice de sedentarismo e sobrepeso em estudantes universitários dos Cursos de Saúde
}

\section{Analysis of dietary profile and sedentary lifestyle index in university students of health courses}

\author{
${ }^{1}$ Thaisnara Priscila Franco Bastos \\ ${ }^{1}$ Josiane Vieira de Souza \\ ${ }^{2}$ Maria de Fátima Alves de Oliveira
}

\section{RESUMO}

O crescimento do índice de sobrepeso, obesidade e sedentarismo na população brasileira vem gerando grande preocupação, visto que, patologias consequentes dessa nova condição alcançaram a principal posição no quadro de mortalidade. Tais índices têm acometido em grande parte a população jovem e adolescente, o que se torna relevante à atuação de pesquisas que visem à prevenção e promoção de saúde a essa faixa etária. O objetivo deste trabalho é analisar o perfil alimentar e o índice de sedentarismo e sobrepeso em estudantes universitários dos cursos de saúde de uma instituição de ensino superior da zona oeste do Rio de Janeiro. A pesquisa foi realizada com 94 estudantes do $1^{\circ}$ período, compreendendo os cursos de Fisioterapia, Educação Física, Enfermagem e Biologia. A coleta de dados foi realizada através de um questionário anexo a um folder contendo informações e dicas sobre alimentação saudável e prática de atividade física regular. O instrumento foi aplicado durante uma palestra sobre Iniciação Cientifica na Instituição. A análise revelou que aproximadamente metade dos universitários apresentavam o quadro de sobrepeso e sedentarismo, além do alto consumo de fast foods e refrigerantes durante a semana. Dados relevantes por se tratar de cursos da área da saúde e possuírem em sua grade de ensino, disciplinas que abordam a temática.

Palavras-chave: sobrepeso; obesidade; sedentarismo; universitários.

\section{ABSTRACT}

The growth rates of overweight, obesity and physical inactivity in the Brazilian population has generated great concern because, consequential pathologies new condition achieved the leading position in the table of mortality. Such indices have largely affected the young and adolescent population, which is relevant to the performance of research aimed at prevention and health promotion in this age group. The objective of this work is to analyze the profile and feed rates of physical inactivity and overweight among university students of health of an institution of higher education west of Rio de Janeiro. The survey was conducted with 94 students in 1st period, comprising courses in Physiotherapy, Physical Education, Nursing and Biology. Data collection was conducted through a questionnaire attached to a folder containing information and tips on healthy eating and regular physical activity. The instrument was administered during a lecture on Scientific Initiation in the institution. The analysis revealed that approximately half of the university presented the condition of overweight and sedentary lifestyle, in addition to the high consumption of fast foods and soft drinks during the week. Relevant data for the case of courses in the area of health and possess in his crate teaching, disciplines that address the topic.

Keywords: University; overweight; obesity; physical inactivity.

1 Graduanda de Fisioterapia - Centro Universitário Moacyr Sreder Bastos.

2 Prof . Orientadora- Centro Universitário Moacyr Sreder Bastos - UniMSB; Centro Universitário de Volta Redonda-UniFOA; LAEFIB/ FIOCRUZ. 


\section{INTRODUÇÃO}

O Brasil vem passando por uma transição, onde historicamente a preocupação era centrada nos níveis elevados de desnutrição e atualmente ela está voltada aos grandes índices de obesidade e sedentarismo (SANTOS e SCHERER, 2010). O aumento de doenças não transmissíveis consequentes dessa nova condição, vem gerando bastante preocupação, visto que, tais patologias ocuparam a principal posição no quadro de mortalidade no Brasil (LINO et al., 2011). Esse quadro tem acometido principalmente jovens e adolescentes, por ser uma fase em que ocorre o aumento da independência, poder de decisão sobre as escolhas de vida e o ritmo de vida corrido, o que consequentemente pode expor o indivíduo, na maioria das vezes, ao sedentarismo e inadequação alimentar (SOUZA et al., 2011).

Os aspectos predisponentes para o aumento do quadro de sedentarismo e sobrepeso nessa faixa da população se dá pelo ritmo de vida acelerado que os jovens possuem. Dupla jornada de trabalho e estudo, pouco tempo para a alimentação balanceada e adequada, acabam por optar por alimentos de preparo rápido ou de fácil acesso, os denominados fast foods. Estudos realizados por Souza et al. (2011) sobre os fatores de risco e de proteção comportamentais relacionados à saúde em jovens e adolescentes, relatam que o estilo de vida dos mesmos não são satisfatórios, abordando o baixo consumo de frutas, inatividade física, o consumo de bebidas alcoólicas e tabaco. Nas ultimas três décadas, de acordo com dados da pesquisa nacional de 2002-2003, sobre tendência nutricional dos adolescentes brasileiros, observa-se o aumento da obesidade em 18 vezes no sexo masculino e 4 vezes no feminino, tornando-se assim uma importante questão de saúde pública a ser tratada (ABBES et al., 2011).

A alimentação inadequada e a inatividade física são fatores relevantes na disseminação da obesidade e de outras doenças crônicas não transmissíveis, e gera impactos pessoais, sociais e financeiros sobre a vida da população e do País (SOUZA et al., 2011). O sedentarismo, desde o século vinte, vem tendo um acréscimo considerável, fato este, preocupante, visto que a atividade física proporciona diversos benefícios à saúde. Os que a praticam regularmente possuem menor risco de desenvolver doenças cardiovasculares, obesidade, ansiedade e depressão, entre outros fatores (ZANCHETA et al., 2010).

A conscientização e a estimulação a prática de hábitos saudáveis, incluindo medidas para a correção do estilo de vida sedentário e o incentivo a alimentação balanceada, precisam estar aderidos aos programas de saúde pública, principalmente dentro de ambientes de ensino (ABBES et al., 2011). Souza et al. (2011) demostram em sua pesquisa, a efetividade da atuação através da elaboração de programas que incentivam uma vida mais saudável. Adultos jovens, especialmente estudantes universitários da área de saúde, possuem importante papel como mediadores e disseminadores dessas informações na sociedade, podendo influenciar o meio onde estão inseridos (NETTO et al., 2012).

O objetivo desse estudo é analisar o perfil alimentar e o índice de sedentarismo e sobrepeso em alunos universitários dos cursos de saúde de uma instituição privada de ensino superior localizada na zona oeste do Rio de Janeiro. O Projeto foi submetido ao comitê de Ética sob número CAEE: 10002313.3.0000.5287.

\section{METODOLOGIA}

Trata-se de um estudo descritivo exploratório, realizado com acadêmicos dos cursos de saúde de uma instituição privada de ensino superior da zona oeste do Rio de Janeiro durante o primeiro semestre de 2013.

Foi utilizado como instrumento de coleta de dados um questionário anexo a um folder contendo informações e dicas sobre alimentação saudável e prática de atividade física, desenvolvido para os acadêmicos da instituição. O questionário possui 8 questões objetivas sobre o perfil alimentar e frequência de atividade física, 
além de uma questão sobre Índice de Massa Corporal (IMC), cuja a tabela estava presente no folder, para que o estudante o calcula-se.

O Instrumento foi aplicado durante uma palestra de Iniciação Científica na instituição, onde foi oferecido aos alunos o folder como forma de promoção a saúde e incentivo a prática de atividade física. Posteriormente o questionário anexo foi destacado pelos mesmos para a análise dos dados. Antes da aplicação do instrumento, os objetivos do estudo foram esclarecidos para que os interessados participassem da amostra. Os dados obtidos na coleta de dados foram tabulados pela plataforma de base de dados do Excel.

\section{RESULTADOS E DISCUSSÃO}

Participaram de pesquisa 94 estudantes, de ambos os sexos, cursando o $1^{\circ}$ período dos cursos de Fisioterapia, Educação Física, Enfermagem e Biologia. Os dados do questionário foram tabulados pela plataforma de base de dados englobando os quatro cursos.

A análise do consumo de frutas, verduras, legumes e grãos apresentou um dado interessante, a maioria dos acadêmicos declarou consumir regularmente esses alimentos; 65 entrevistados declararam consumir três vezes ou mais durante a semana, como se pode observar na Figura 1. O que contrapõe o estudo de Souza et al., (2011) e Ferreira et al., (2011), que relatam em suas pesquisas o baixo consumo dos mesmos por parte de jovens e adolescentes.

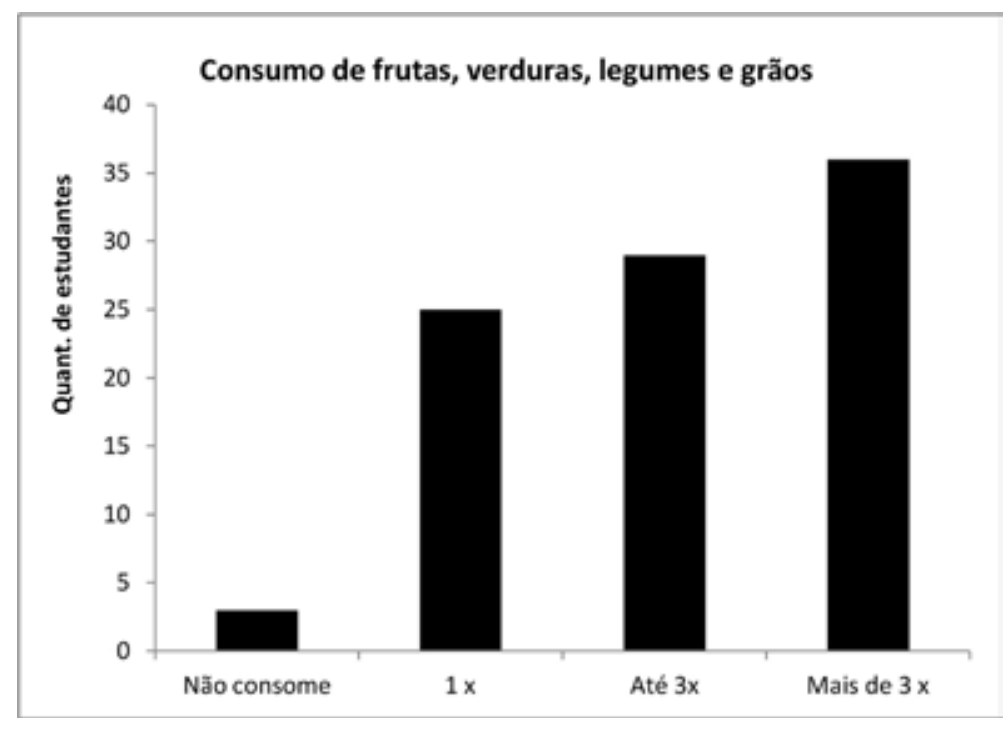

Figura 1: Consumo de frutas, verduras, legumes e grãos durante a semana

A ingesta regular desses alimentos trás diversos benefícios, pois eles possuem poucas calorias, e diversos micronutrientes. Além de atuar diretamente na ajuda à prevenção de doenças como o câncer, diabetes, hipertensão, doenças ósseas, cardiovasculares, inflamatórias e intestinais. Demais estudos se fazem sobre a funcionalidade desses alimentos, e para tais benefícios é necessário o consumo balanceado e regular (VIDAL et al., 2012).

Em contraponto, o consumo de fast foods se revelou alto, como apresentado no gráfico da Figura 2. Cerca de aproximadamente 60 acadêmicos consomem hambúrgueres, salgados, pizza e chocolate presentes em sua dieta, três vezes ou mais durante a semana. 


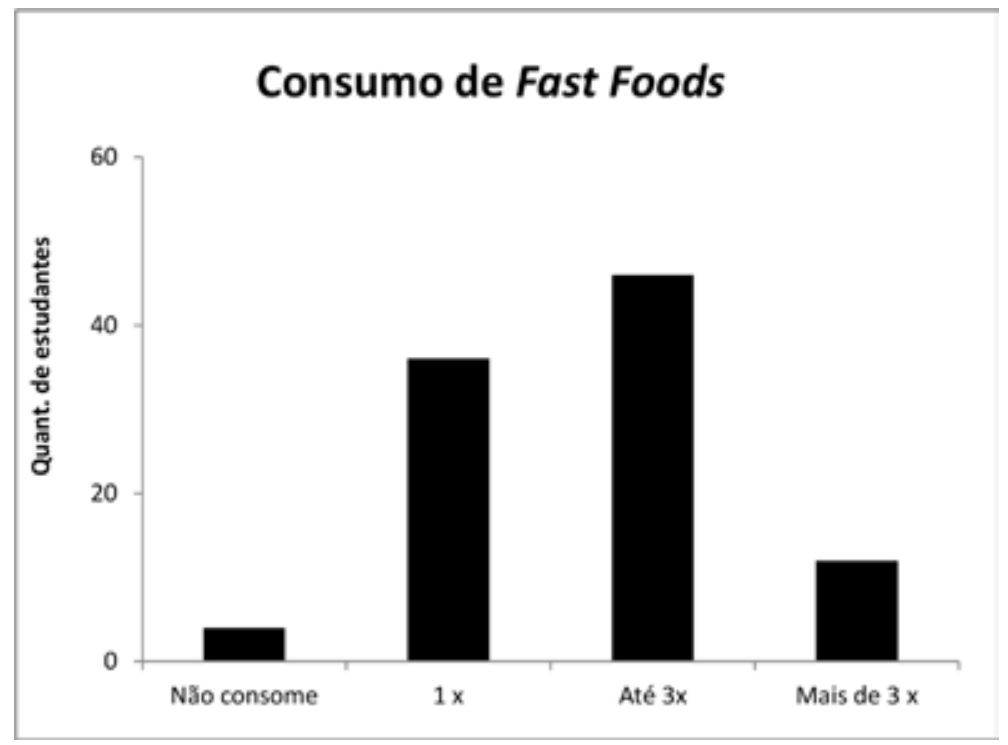

Figura 2: Consumo de Fast Foods durante a semana

Segundo uma reportagem na Revista Carta Capital (2012), sobre os crescentes índices de obesidade no mundo, as pessoas estão passando cada vez menos tempo cozinhando, e mais tempo preocupadas com seu trabalho e atividades exteriores ao lar. É o que nos remete ao gráfico anterior, devido a grande quantidade de estudos, ou dupla jornada entre trabalho e faculdade, a preferencia pela ingestão de alimentos de rápido preparo ou acesso é alta. Além disso, deve-se considerar a herança cultural imposta, passada de pais para filhos. A atenção à alimentação adequada deve ser inserida desde a infância, observando que hábitos adquiridos durante esta fase e adolescência tendem a ser perpetuados por toda vida (SOUZA et al., 2011).

Atualmente, o consumo de fast foods é um quadro comum observado em jovens e adolescentes. Com as facilidades da vida moderna, a incidência de maus hábitos alimentares entre os jovens tem crescido. É comum vê-los se alimentando em lanchonetes e cantinas escolares onde o cardápio costuma ser repleto de comidas ricas em gordura e bebidas nada saudáveis, como refrigerantes e sucos artificiais (FERREIRA et al., 2012).

Em concordância com Ferreira et al., (2012), a análise do consumo de refrigerante apresentado pelos acadêmicos participantes da amostra também foi elevado (Figura 3). Cerca da metade dos universitários (47) que responderam o questionário declararam consumir três vezes ou mais refrigerantes durante a semana.

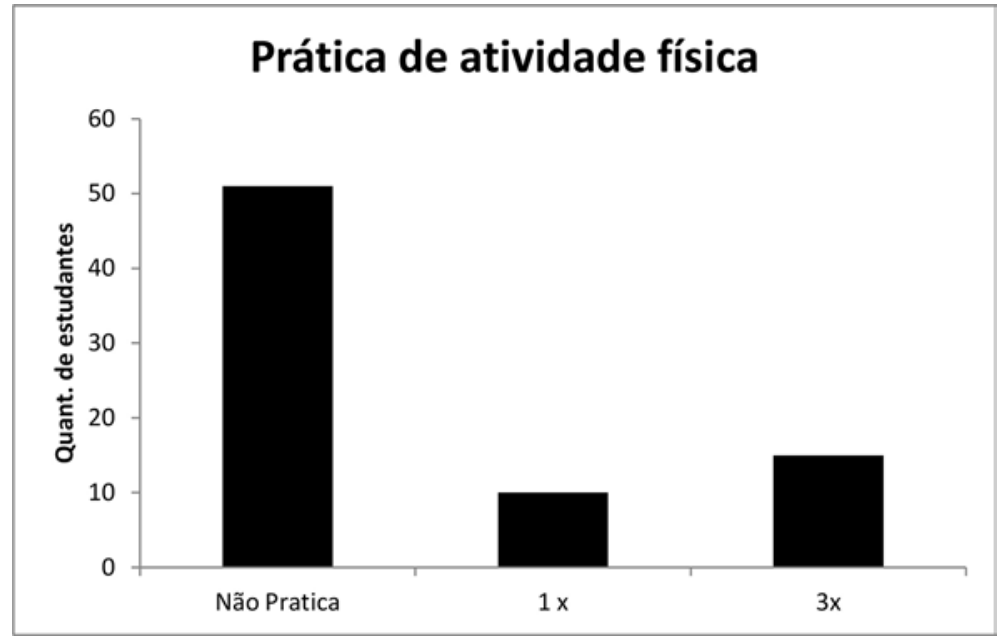

Figura 3: Consumo de de refrigerante 
O consumo exagerado de refrigerante acarreta diversos malefícios à saúde. Dentre eles podemos destacar a grande densidade energética, pois o consumo de produtos sob forma líquida tem efeitos fisiológicos diferentes em relação à forma sólida, em relação à líquida, a sensação de saciedade é menor, fazendo com que ocorra a ingestão dos alimentos calóricos de forma sólida resultando no consumo de mais calorias (ESTIMA et al., 2011).

Em relação à análise da quantidade refeições diárias (Figura 4), a presente pesquisa apresentou que a maioria dos participantes (42) se alimentava em média três vezes ao dia. Dado este que vem a complementar os valores anteriores. O habito de omitir refeições gera o aumento do consumo de refeições rápidas, sendo considerados comportamentos diretamente relacionados com o sobrepeso e a obesidade (FERREIRA et al., 2012).

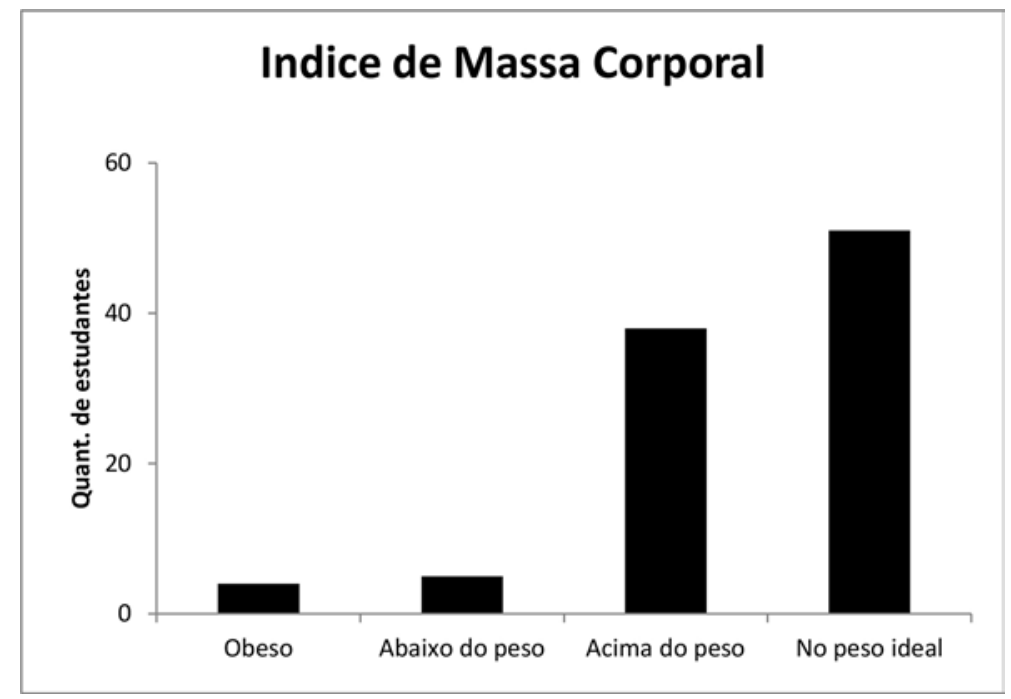

Figura 4: Quantidade de refeições ao dia

O Ministério da Saúde (2013) estabelece pelo menos seis refeições diárias, sendo elas: café da manhã, almoço e jantar e três lanches saudáveis por dia, sem pular as refeições. Isso implicará em maior saciedade e menor procura por alimentos ricos em gorduras e açucares, além de acelerar o metabolismo e consequente gasto energético.

Dentre os dados apurados na análise, o que mais destacou foi o elevado índice de sedentarismo em universitários dos cursos de saúde, principalmente do curso de Educação Física (Figura 5), que possui diretamente em sua grade de ensino e de estágio essa prática regular de atividade física.

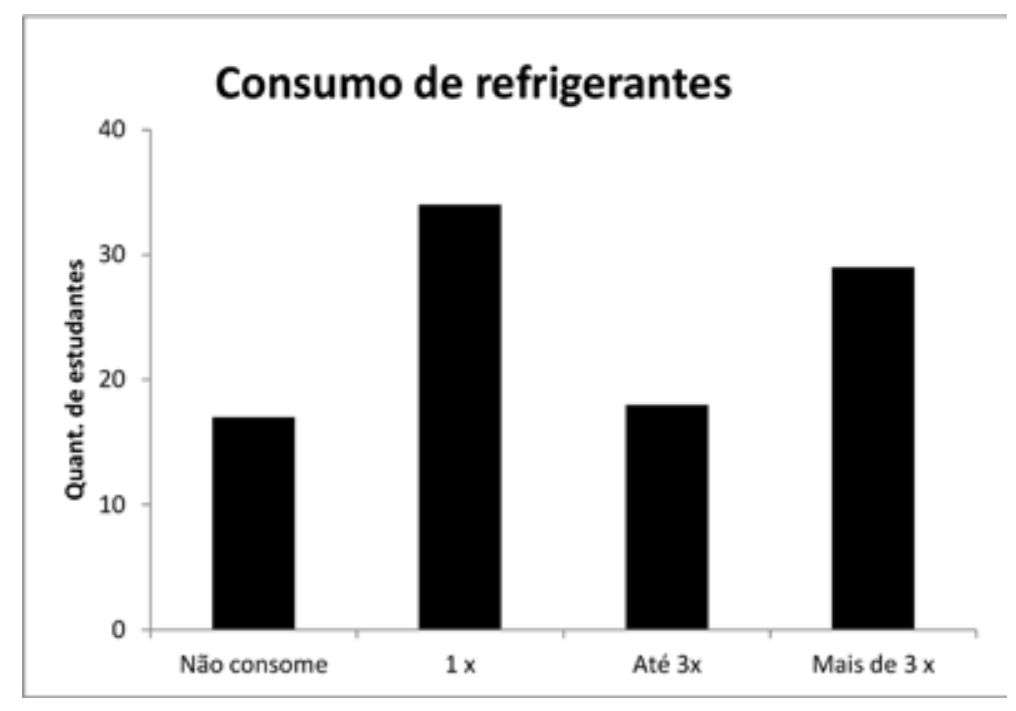

Figura 4: Frequencia de ativdade física semanal 
Segundo a análise de dados, cerca de mais da metade dos acadêmicos entrevistados (51) declararam não praticar nenhuma atividade física. Fator preocupante, visto que a faculdade é uma instituição de produção e multiplicação do conhecimento. É através dos futuros profissionais dos cursos direcionados a saúde, que os hábitos saudáveis devem ser incentivados, visto que são eles os responsáveis pela promoção e atuação ao setor primário da saúde (NETTO et al., 2012).

A atividade física e a alimentação são dois comportamentos considerados prioritários para a promoção da saúde e prevenção de doenças crônicas não degenerativas, como diabetes e doenças cardiovasculares em populações contemporâneas (TEIXEIRA et al., 2012). O estilo de vida, no qual a maior parte do tempo livre é passada em frente à televisão, utilizando computadores, é um dos responsáveis pelo sedentarismo. Se o consumo de alimentos ricos em gorduras e açucares está elevado, e o indivíduo não queimar as calorias excedentes, acarretará em sobrepeso e consequente obesidade e hipertensão, contribuindo para mortes decorrentes de doenças cardiovasculares em indivíduos cada vez mais jovens (FERREIRA et al., 2012).

Na ultima análise, pode-se observar como consequência, a grande prevalência de sobrepeso nos universitários. Aproximadamente metade dos acadêmicos declarou estar acima do peso, como observado na Figura 6.

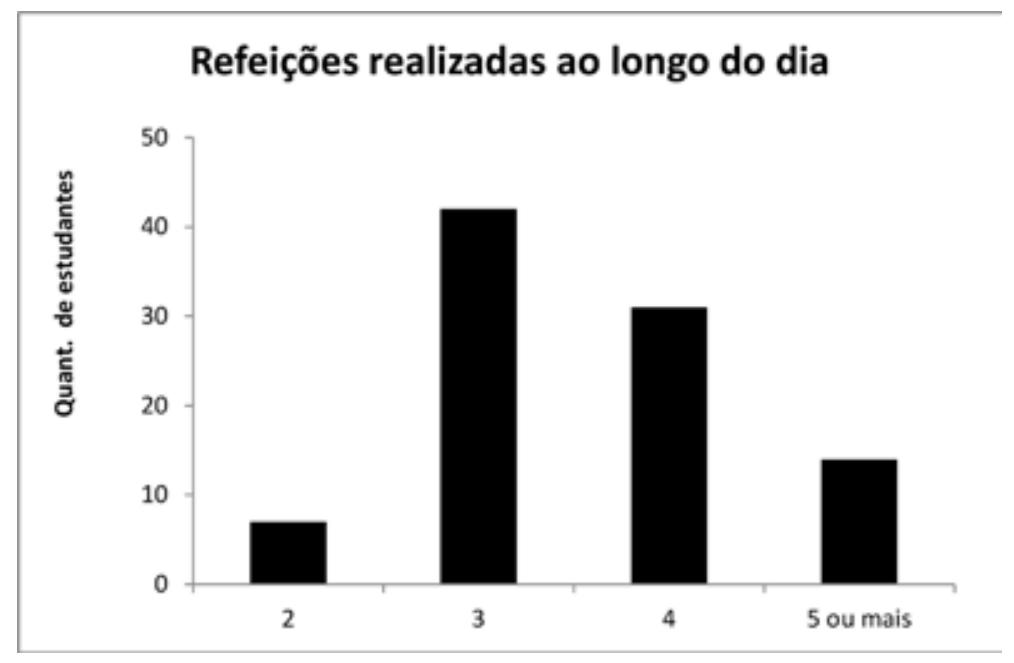

Figura 6: Índice de massa corporal

Os dados foram obtidos através do calculo do Índice de Massa Corporal (IMC), presente no folder distribuído para os acadêmicos. Corroborando com os dados anteriores apresentados, a análise indicou os altos índices de sobrepeso. Fato que vem a confirmar que a alimentação desregrada associada ao sedentarismo oferece como consequência o aumento de peso e a obesidade, sujeitando o indivíduo a propensão de várias doenças decorrentes desse quadro (SEBOLD et al., 2011).

O acúmulo de gordura corporal exerce várias consequências sobre a mortalidade e morbidade na vida do indivíduo. Além disso, as consequências da obesidade são responsáveis por causar diversos gastos com a saúde publica e promover a conscientização sobre práticas saudáveis, contribuirá para a redução desses gastos segundo Rodrigues et al., 2011 e Souza et al., 2011.

Embora haja o incentivo da mídia para o consumo de fast foods e alimentos industrializados, atualmente se observa também uma preocupação com o bem estar e qualidade vida presentes nesses meios, visto o quadro grave em que a população se insere. 


\section{CONCLUSÃO}

O quadro de sedentarismo e sobrepeso são fatores extremamente preocupantes na sociedade atual, e fator desencadeador de diversas doenças e disfunções em faixas etárias cada vez mais jovens, inclusive ao óbito. Este estudo, concluiu que até mesmo indivíduos que tem acesso a informações sobre saúde, alimentação adequada e prática de atividade física regular, não a fazem. Em concomitância com a população jovem em geral, onde o consumo de fast foods, alimentos industrializados, ricos em gorduras e sódio tem sido extremamente elevado.

Em relação ao consumo de frutas, verduras e grãos, a análise se mostrou satisfatória inicialmente, visto que a maioria dos participantes da amostra declara consumi-los regularmente. Porém o consumo de tais alimentos, de forma isolada, não proporciona os benefícios à saúde, se acompanhado de alta ingestão de frituras, refrigerantes, sódio e tudo que se inserir nesse âmbito. Associado a isso, a prática de atividade física regular, pobre ou nula, proporcionando o elevado sobrepeso presente na análise do Índice de massa corporal desses jovens.

É evidente, a importância da inserção de medidas que promovam o incentivo a qualidade de vida e hábitos saudáveis, principalmente em acadêmicos da classe de saúde, onde através dos mesmos, tais informações se multiplicaram e dissiparam sobre a população. No entanto, mais estudos são necessários, em ambientes universitários, com o objetivo da atuação para acadêmicos e por acadêmicos desses cursos, visando o incentivo a prática de atividade física regular e alimentação saudável. O presente estudo foi resultados de um desses instrumentos de promoção à saúde, um folder, repassado aos alunos de Fisioterapia, Educação Física, Enfermagem e Biologia com o proposito de promover mudanças de hábitos e abranger á área de conhecimento. Este estudo contribuiu para que novas pesquisas no âmbito universitário, em relação à alimentação saudável e a prática de atividade física possam ser realizadas.

\section{AGRADECIMENTOS}

Ao Centro Universitário Moacyr Sreder Bastos- UniMSB que contribuiu financeiramente para o desenvolvimento do Projeto de Iniciação Cientifica, assim como a disponibilização do local para a palestra e coleta de dados. 


\section{REFERÊNCIAS}

ABBES, Priscila Trapp; LAVRADOR, Maria Silvia Ferrari; ESCRIVÃO, Maria Arlete Meil Schimith; et al. Sedentarismo e variáveis clínico-metabólicas associadas à obesidade em adolescentes. Revista de Nutrição, Campinas, v.24, n.4, p.529-538, Jul/Ago., 2011.

ESTIMA, Camilla Chermont P; PHILIPPI, Sonia Tucunduva; ARAKI, Erica Lie et al. Consumo de bebidas e refrigerantes por adolescentes de uma escola pública. Revista Paulista de Pediatria. v.29 , n.1, p.41-45, São Paulo, 2011.

FERREIRA, Talita da Silva; CHAFAUZER Caroline; JÚNIOR, Fernando Moreira de Araújo, et al. Obesidade central em jovens. Revista Science in Health. v.3, n.2, p. 61-73, 2012.

LINO, Marina Zago Ramos; MUNIZ, Pascoal Torres; SIQUEIRA, Kamile Santos. Prevalência e fatores associados ao excesso de peso em adultos: inquérito populacional em Rio Branco, Acre, Brasil, 2007-2008. Caderno de Saúde Pública, Rio de Janeiro, v.27, n.4, p.191-810, Abr, 2011.

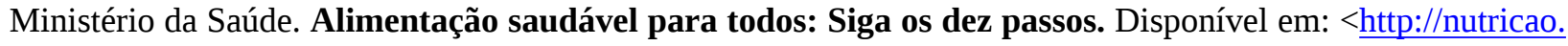
saude.gov.br/>. Acesso em 27/11/13.

NETTO. Raquel Simões Mendes; SILVA, Camilla Santos da; COSTA, Dayanne et al. Nível de atividade física e qualidade de vida de estudantes universitários da área de saúde. Revista Brasileira de Ciências da Saúde, ano 10, no 34, out/dez, 2012.

Revista Carta Capital. Um problema de peso. Ano 23. n.729, Dezembro, 2012.

RODRIGUES, Paula Afonso; MARQUES, Maria Helena; CHAVES, Maria das Graças Afonso Miranda; et al. Prevalência e fatores associados a sobrepeso e obesidade em escolares da rede pública. Ciência \& Saúde Coletiva, v. 16, n.1, p. 1581-1588, 2011.

SANTOS, Andreia Mendes dos; SCHERER, Patrícia Teresinha. Reflexões acerca das políticas públicas no enfrentamento a obesidade no Brasil. Revista Sociedade em Debate, Pelotas, v. 17, n.1, p. 219-236, Jan.-Jun, 2011.

SEBOLD, Luciara Fabiane; RADUNZ, Vera; CARRARO,Telma Elisa. Percepção sobre cuidar de si, promoção da saúde e sobrepeso entre acadêmicos de Enfermagem. Esc Anna Nery (Imp.), v. 15, n. 3, p.536-541, Jul-Set, 2011.

SOUZA, Evanice Avelino de; BARBOSA FILHO, Valter Cordeiro; NOGUEIRA, Júlia Aparecida Devidé; et al., Atividade física e alimentação saudável em escolares brasileiros: revisão de programas de intervenção. Caderno de Saúde Pública, Rio de Janeiro, v.27, n.8, p.1459-1471, Ago, 2011.

TEIXEIRA, Amanda Solimani; PHILIPPI, Sonia Tucunduva; LEAL, Greisse Viero da S.; et al. Substituição de refeições por lanches em adolescentes. Revista Paul Pediatr, v.30, n. 3, p.330-337, 2012.

VIDAL, Andressa Meirelles; DIAS, Danielle Oliveira; MARTINS, Emanuelle Santana Melo; et al. A ingestão de alimentos funcionais e sua contribuição para A diminuição da incidência de doenças. Cadernos de Graduação - Ciências Biológicas e da Saúde, v. 1, n.15, p. 43-52, Out. Aracaju, 2012.

ZANCHETA, Luane Margareth; BARROS, Marilisa Berth de Azevedo; CÉZAR Chester Luiz Galvão; et al. Inatividade física e fatores associados em adultos, São Paulo, Brasil. Revista Brasileira de Epidemiologia, São Paulo, v13 n.3, p.387-99, 2010. 